\title{
Editorial
}

\author{
Hervé Bourlard \\ IDIAP, Rue du Simplon 4, CH- 1920 Martigny, Switzerland \\ Email:bourlard@idiap.ch
}

\section{loannis Pitas}

Department of Informatics, Aristotle University of Thessaloniki, Thessaloniki, TK 54006, Greece

Email: pitas@zeus.csd.auth.gr

\section{Kenneth Kin-Man Lam}

Centre for Multimedia Signal Processing, Department of Electronic and Information Engineering,

The Hong Kong Polytechnic University, Hong Kong

Email:enkmlam@polyu.edu.hk

\section{Yue Wang}

The Bradley Department of Electrical and Computer Engineering, Virginia Polytechnic Institute and State University, VA 24061-0111, USA

Email: yuewang@vt.edu

Biometric signal processing is an emerging technology that enables the authentication, identification, or verification of an individual based on physiological, behavioral, and molecular characteristics. With the advancement of computer vision and pattern recognition techniques, together with highspeed computers, research related to biometrics has developed rapidly in the last several decades, and has led to various applications. Biometric techniques include recognizing faces, hands, voices, signatures, irises, fingerprints, DNA patterns, and so forth. These enabling technologies for biometrics will play an important role in security, smart card, and personalized eCommerce applications. The analysis of biometric information is a challenging task, and a wide range of signal processing techniques has to be applied. The success of the applications relies heavily on the efficiency, reliability, and accuracy of these biometric signal processing techniques.

This special issue brings together researchers working on biometric signal processing and its applications, with a particular emphasis on person authentication and identification. In this special issue, we are pleased to present new techniques as well as developments of the different signal processing techniques and their applications. We have three papers on speaker verification, two papers on fingerprint matching, four papers on human face detection and recognition, two papers on signature verification, and one paper on gait recognition.

Speaker verification and fingerprint pattern recognition are among the very first applications in biometric signal processing. The first paper by Bimbot et al. is a tutorial paper that provides an overview of a state-of-the-art textindependent speaker verification system. A modular scheme of the training and test phase of the system is introduced. Gaussian mixture model and ceptral analysis, which are the dominant techniques for speaker verification, are explained in detail in this paper. Other speaker modeling alternatives, scoring normalization, and the extension of speaker verification techniques to other applications are also covered. This is a useful paper for researchers working in this field. The second paper by Mak et al. considers a new channel compensation approach to telephone-based speaker verification. This direction of speaker verification has attracted much attention recently because of the proliferation of eBanking and eCommerce, which require the verification of a speaker over the telephone. The paper proposes to combine a handset selector with stochastic feature transformation to reduce the distortion caused by the limited bandwidth of the telephone network. In addition, a divergence-based handset selector with out-of-handset rejection capability to identify the unseen handsets is proposed. The last paper on speaker verification is by Besacier et al., and it presents the investigation of speaker verification over the Internet at the protocol level and at the speech signal level. At the protocol level, the paper recommends the transmission of data models or features instead of raw biometric data in order to reduce the transmission time, and the use of encryption/decryption for enhancing data security. At the signal level, the paper shows that packet 
loss is not a major problem for text-independent speaker authentication. However, the use of a low bit rate coder will greatly degrade the performance. The next two papers are on fingerprint segmentation and recognition. The paper by Chen et al. proposes a novel algorithm for the block featurebased segmentation of fingerprints. Its major contribution is an integrative approach to feature analysis and segmentation. Adding morphological postprocessing, the method could significantly improve the quality of segmenting fingerprints with greatly reduced misclassification. The paper by Yin et al. focuses on an accurate estimation of the ridge distance in fingerprint feature analysis. Its major contribution is a balanced effort on both algorithm development and performance evaluation. Due to the lack of much published work on this topic, the paper could significantly motivate fertile scientific discussions.

Research on human face recognition has been growing rapidly over the last two decades. To identify a person in an open environment, human face recognition is the most natural approach. This is because to collect useful data for recognition, face recognition has the advantage of being nonintrusive, requiring little cooperation from the person being identified. The paper by Jiang addresses the issues of detecting human faces in a complex airport environment. The paper presents a new variant of the AdaBoost to detect human faces, namely, S-AdaBoost, which uses the AdaBoost distribution weight as a dividing tool to separate the input face space into inlier and outlier face spaces; dedicated classifiers are then used to handle the inliers and outliers in their corresponding face spaces. This is an effective approach to locating human faces in a complex background. The accuracy of detecting a human face and locating its respective facial features has a direct impact on the performance of the face recognition algorithms to be used. The next three papers are also on face recognition. The paper by Perronnin et al. proposes a novel approach to face recognition by modeling the transformation between face images of the same person. The transformation is approximated by means of a collection of local transformations with a constraint to make neighboring transformations consistent with each other. Local transformations and neighboring constraints are embedded within a probabilistic framework using 2D hidden Markov models (HMMs). Another major contribution of this paper is the introduction of the Turbo-HMM, which is an efficient technique to approximate intractable 2D HMMs. The next two papers on face recognition consider the optimal conversion of a color image to a monochromatic image and the combination of different face recognition results, respectively, to improve face recognition performance, instead of considering a new face recognition algorithm. The paper by Jones III et al. proposes optimal methods to convert a color image to a monochromatic image for face recognition. Actually, this issue has not been considered in the current face recognition algorithms. Three approaches-Karhunen-Loève analysis, the linear regression of color distribution, and a genetic algorithm-are explored to determine the optimal conversion. The color-conversion methods are independent of the face recognition approach being used, but can improve its

recognition performance. The other paper by Huang et al. presents a way to achieve a better recognition performance level by combining the classifier outputs based on different face recognition techniques. The paper proposes three methods to combine the classifiers, namely, the normalization of the classifier output, the selection of classifier(s) for recognition, and the weighting of each classifier.

Signature verification is also a commonly used biometric identification technique. Signatures have been widely used in bank and credit card transactions as a means of authentication, and most computers or hand-held devices are also equipped with I/O to allow handwriting input. In addition, a signature or a piece of handwriting may be changed by the user, but that is impossible with fingerprints, face, irises, and so forth. This special issue has two papers on signature and handwriting verification. The paper by Vielhauer and Steinmetz presents an approach to derive biometric hashes based on handwriting. The paper investigates the degree to which each of the statistical feature parameters contributed to the overall intrapersonal stability and interpersonal value space. A feature correlation method for feature analysis and selection is also proposed. The next paper by Coetzer et al. is on offline signature verification. The paper proposes to use the discrete Radon transform first to extract global features of a scanned signature, and then to feed the features into the HMM in order to model the signature. Most of the existing signature verification approaches utilize local features. It is likely that the algorithm proposed in the paper can be incorporated with other existing signature verification methods based on local features to achieve a significant improvement.

The last paper in this issue is by BenAbdelkader et al. They study human identification at a distance using gait recognition. This research has recently attracted growing interest from computer vision researches even though it is still at its infancy. The paper describes a novel gait recognition technique based on the image self-similarity of a walking person. The major advantages of the method are that it is correspondence-free, works well with low-resolution video, and is robust to variation in clothing, lighting, and to segmentation errors.

In summary, this special issue presents a wide range of different biometric features and techniques for applications, such as speaker verification, fingerprint recognition, face recognition, signature verification, and gait recognition. We can foresee that developments in this field will become even more rapid in the future. We hope that the techniques presented in this issue will be of great use to researchers in this field and will provide them with possible directions for the development of biometric technologies. We wish to thank all the authors for their contributions and all the reviewers for their diligent efforts in evaluating and commenting on the papers.

Hervé Bourlard
Ioannis Pitas
Kenneth Kin-Man Lam
Yue Wang

Hervé Bourlard Man Lam 
Hervé Bourlard received the Electrical and Computer Science Engineering degree and the Ph.D. degree in applied sciences both from Faculté Polytechnique de Mons, Belgium. After having been a member of the scientific staff at the Philips Research Laboratory of Brussels and an R\&D Manager at L\&H SpeechProducts, he is now Director of the IDIAP Research Institute and Professor at the Swiss Federal Institute of Technology

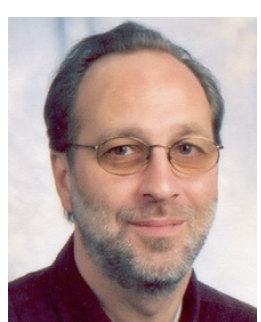
at Lausanne (EPFL), and Director of a National Center of Competence in Research in "Interactive Multimodal Information Management." He is also an External Fellow of the International Computer Science Institute (ICSI) in Berkeley, Calif, and a member of the ICSI Board of Trustees. H. Bourlard is the author/coauthor of 2 books and over 180 reviewed papers (including one IEEE paper award) and book chapters. He is an IEEE Fellow for contributions in the fields of statistical speech recognition and neural networks. He is (or has been) a member of the program and/or scientific committee of numerous international conferences (e.g., General Chairman of IEEE Neural Networks Signal Processing 2002, General Chairman of Eurospeech'2003) and journals, and past Coeditor-in-Chief of the Speech Communication journal. His main interests are in signal processing, statistical pattern classification, multi-channel processing, artificial neural networks, and applied mathematics, with applications to speech processing, speech and speaker recognition, language modeling, computer vision, and multimodal processing.

Ioannis Pitas received the Diploma of Electrical Engineering in 1980 and the Ph.D. degree in electrical engineering in 1985 , both from the University of Thessaloniki, Greece. Since 1994 he has been a Professor at the Department of Informatics, University of Thessaloniki, Greece. His current interests are in the areas of digital image processing, multimedia signal processing, multidimensional signal processing, and computer vi-

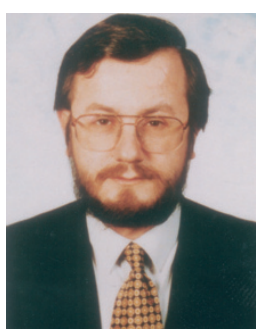
sion. He has published over 450 papers, contributed in 17 books and authored, coauthored, edited, or coedited 7 books in his area of interest. He is the coauthor of the books Nonlinear Digital Filters: Principles and Applications (Kluwer, 1990) and 3D Image Processing Algorithms (Wiley 2000). He is the author of the books Digital Image Processing Algorithms (Prentice Hall, 1993), Digital Image Processing Algorithms and Applications (Wiley 2000), Digital Image Processing (in Greek, 1999). He is the editor of the book Parallel Algorithms and Architectures for Digital Image Processing, Computer Vision and Neural Networks (Wiley, 1993) and coeditor of the book Nonlinear Model-Based Image/Video Processing and Analysis (Wiley 2000). He is a principal investigator/researcher in more than 40 competitive R\&D projects and in 11 educational projects, all mostly funded by the European Union. He is Associate Editor of the IEEE Transactions on Circuits and Systems, IEEE Transactions on Neural Networks, IEEE Transactions on Image processing, IJIG, IEICE, Circuits Systems and Signal Processing (CSSP), coeditor of Multidimensional Systems and Signal Processing, member of the editorial board of 6 journals and guest editor in 6 special journal issues. He was Chair of the 1995 IEEE Workshop on Nonlinear Signal and Image Processing (NSIP95). He was Technical Chair of the 1998 European Signal Processing Conference. He was the General Chair of IEEE ICIP2001. He was Cochair of the 2003 International workshop on Rich media content production. He was Technical Cochair of the 2003 Greek Informatics conference (EPY).
Kenneth Kin-Man Lam received his Associateship in electronic engineering from The Hong Kong Polytechnic University (HKPolyU) in 1986, his M.S. degree in communication engineering from the Imperial College of Science, Technology and Medicine in 1987, and his Ph.D. degree from the Department of Electrical Engineering, University of Sydney in 1996. From 1990 to 1993, he was a Lecturer at the De-

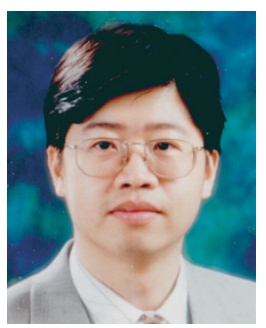
partment of Electronic Engineering of HKPolyU. He joined the Department of Electronic and Information Engineering, HKPolyU again as an Assistant Professor in October 1996, and has become an Associate Professor since February 1999. Dr. Lam was actively involved in professional activities. In particular, he was the Secretary of the 2001 International Symposium on Intelligent Multimedia, Video and Speech Processing, a Program Committee Member of the 2002 Conference on Visual Communications and Image Processing, and the Secretary of the 2003 IEEE International Conference on Acoustics, Speech, and Signal Processing. Currently, Dr. Lam is the Treasurer of the IEEE Hong Kong Chapter of Signal Processing, a Program Committee Member of the 2004 Conference on Advanced Concepts for Intelligent Vision Systems, and the Technical Chair of the 2004 International Symposium on Intelligent Multimedia, Video and Speech Processing (ISIMP 2004) to be held in Hong Kong in October 20-22, 2004. His research interests include face recognition, image and video processing, and computer vision.

Yue Wang received his Ph.D. degree in electrical engineering from the University of Maryland in 1995. He is currently an Associate Professor of electrical and computer engineering at the Virginia Polytechnic Institute and State University (Virginia Tech), Alexandria, Va. He is also affiliated with the Johns Hopkins Medical Institutions, Baltimore, MD, as an Adjunct Associate Professor of radiology. His research interests focus on computational bioinformatics and bioimaging.

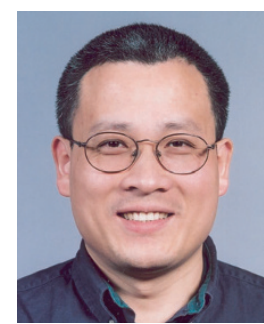

